\title{
Induction of potent antitumor immunity by in situ targeting of intratumoral DCs
}

\author{
Katsuyoshi Furumoto, Luis Soares, Edgar G. Engleman, and Miriam Merad \\ Department of Pathology, Stanford University School of Medicine, Palo Alto, California, USA.
}

\begin{abstract}
Recent reports of tumor regression following delivery of autologous tumor antigen-pulsed DCs suggest that defective antigen presentation may play a key role in tumor escape. Here we show in two different murine tumor models, CT26 (colon adenocarcinoma) and B16 (melanoma), that the number and activation state of intratumoral DCs are critical factors in the host response to tumors. We used CCL20/macrophage inflammatory protein-3 $\alpha$ (MIP-3 $\alpha)$ chemokine to increase the number of tumoral DCs and intratumoral injections of CG-rich motifs (CPGs) to activate such cells. Expression of CCL20 in the tumor site attracted large numbers of circulating DCs into the tumor mass and, in the case of CT26 tumors, led to complete tumor regression. Intratumoral CPG injections, in addition to CCL20, were required to induce therapeutic immunity against B16 tumors. In this model CPG overcame tumor-mediated inhibition of DC activation and enabled tumoral DCs to cross-present tumor antigens to naive CD8 $\mathrm{T}$ cells. CPG activation of tumoral DCs alone was not sufficient to induce tumor regression in either tumor model, nor was systemic delivery of the DC growth factor, Flt3 ligand, which dramatically increased the number of circulating DCs but not the number of tumoral DCs. These results indicate that the number of tumoral DCs as well as the tumor milieu determines the ability of tumor-bearing hosts to mount an effective antitumor immune response. Our results also suggest that DCs can be manipulated in vivo without delivery of defined tumor antigens to induce a specific $T$ cell-mediated antitumor response and provide the basis for the use of chemokines in DC-targeted clinical strategies.
\end{abstract}

\section{Introduction}

The ability of tumors to escape the immune system has been a major obstacle to the development of effective tumor immunotherapy. Both central and peripheral immune tolerance have been implicated in the failure of tumor-bearing hosts to mount an immune response to their tumors. Central tolerance may play a fundamental role in the lack of response against self-tumor-associated antigens (Ag's), while peripheral tolerance may explain the lack of response against tumor-specific Ag's.

DCs are believed to play a critical role in antitumor immune responses. These cells are the most potent APCs known, uniquely capable of inducing immunity to newly introduced Ag's $(1,2)$. Normally, DCs reside as immature cells in peripheral tissues where they sample the environment by taking up and processing local Ag's. In the presence of certain toll-like receptor ligands, these cells not only take up and process Ag's but also undergo activation and maturation and then migrate to the draining LNs where they prime specific CD4 and CD8 T cells to these Ag's. The presence in a wide range of tumors of immature DCs that are unable to stimulate $\mathrm{T}$ cells (3-9) suggests a possible role for these cells in the failure of tumor-bearing hosts to mount an effective antitumor response. This view is supported by a recent study in melanoma patients that showed that tumor-associated DCs are present mostly at the periphery of tumors and express low levels of costimulatory molecules, while the majority of $\mathrm{T}$ cells infiltrating tumors have a naive phenotype (10). The presence of naive but not memory or

Nonstandard abbreviations used: antigen (Ag); bone marrow (BM); CG-rich motif (CpG); complete medium (CM); control oligonucleotide (ODN-CTR); effector/target (E/T); Fms-like thyrosine kinase 3 ligand (Flt3L); Langerhans cell (LC); macrophage inflammatory protein- $3 \alpha$ (MIP-3 $\alpha)$; mean fluorescence intensity (MFI); T cell receptor (TCR).

Conflict of interest: The authors have declared that no conflict of interest exists. Citation for this article: J. Clin. Invest. 113:774-783 (2004). doi:10.1172/JCI200419762. effector T cells in tumors may be related to the failure of tumoral DCs to provide an adequate stimulus or possibly to the induction of $\mathrm{T}$ cell tolerance by the immature DCs. By contrast to tumoral DCs, in vitro-generated DCs can induce an effective T cell-mediated antitumor immune response in vivo (11), indicating that the $\mathrm{T}$ cells of tumor-bearing hosts are capable of recognizing and responding to tumor Ag's and suggesting again that the tumor milieu prevents tumoral DCs from inducing an effective immune response. Indeed, injection of immature Ag-pulsed DCs can induce a specific tolerogenic response, while similarly pulsed DCs, when matured, induced a typical Th1 immune response (12).

Based on the recognition of the central role of DCs in initiating immune responses, a variety of strategies have been devised to use DCs to stimulate immunity against tumor Ag's. Most of these strategies rely on the activation and maturation of DCs ex vivo and their subsequent reinfusion to tumor-bearing recipients after a pulse with tumor Ag's expressed as peptides, protein, or nucleic acids (11). Ex vivo manipulation of DCs, however, is time consuming and costly, requires the use of numerous cytokines, and exposes the patient to increased risk of infection. To avoid manipulation of DCs in vivo, we developed an approach to expand, load, and activate DCs in vivo. By administering a DC growth factor, Fms-like thyrosine kinase 3 ligand (Flt3L), to tumor-bearing mice, followed by subcutaneous injection of oligodeoxynucleotides containing unmethylated CG-rich motifs (CpGs) along with a defined tumor $\mathrm{Ag}$, we were able to induce significant antitumor responses in mice challenged with B16 melanoma (13). The induction of a strong and durable immune response was dependent on the accumulation in skin of high numbers of Flt3L-mobilized DCs, which facilitated their loading and activation with a local injection of a mixture of tumor Ag and CpG. These results suggested that access of DCs to tumor Ag's, as well as the ability of these cells to mature, are critical for the induction of an efficient immune response. This view is sup- 
ported by a recent study that showed that even when DCs are engineered to express high levels of tumor Ag's in vivo, DC expansion and activation were required for the induction of antitumor immunity (14). In both studies, however, therapy was dependent on Flt3L treatment and vaccination with a defined tumor Ag.

In the current study we attempted to increase the uptake of tumor Ag's by DCs in vivo by directing circulating DCs to tumors rather than delivering exogenous tumor Ag's to Flt3L-mobilized DCs. To do so, we used CCL20/macrophage inflammatory protein-3 $\alpha$ (MIP-3 $\alpha$ ) chemokine, a potent chemoattractant for a subset of DCs in both humans and mice (15-17). This technique allowed us to determine if the number of DCs in the tumor site is a critical factor in the induction of antitumor immunity. We also used CPG to activate tumoral DCs and evaluate the effect of the maturational state of these cells on the development of antitumor immunity. By using either CCL20-transduced tumor cells or CCL20 protein injections into the tumors in the presence or absence of local CPG injections, we sought to explore the biology and the immune targets of each of these molecules in vivo and asked whether they can synergize to induce a therapeutic antitumor response.

\section{Methods}

Animals. Six- to ten-week-old male C57BL/6 and BALB/c mice were purchased from The Jackson Laboratory (Bar Harbor, Maine, USA). Generated as described (18) and purchased from The Jackson Laboratory, $1100 \mathrm{Mjb}$ mice express an $\mathrm{H}-2 \mathrm{~K}^{\mathrm{b}}$-restricted $\mathrm{T}$ cell receptor (TCR) specific for the octamer peptide. $\mathrm{CD}^{-/-}$and $\mathrm{CD}^{-/-}$mice were generated as described $(19,20)$ and were also purchased from The Jackson Laboratory. All mice were housed in the Stanford animal facility in accordance with the NIH guidelines.

Cytokines and media. Recombinant human Flt3L, murine CCL20, murine GM-CSF, and IL-4 were purchased from PeproTech Inc. (Rocky Hill, New Jersey, USA). Cytokines and chemokines were reconstituted in PBS. Cells were cultured in vitro in complete medium (CM), which included RPMI-1640 supplemented with $10 \%$ FCS (BioWhittaker Inc., Walkersville, Maryland, USA), penicillin G (100 $\mathrm{U} / \mathrm{ml})$, and streptomycin $(100 \mu \mathrm{g} / \mathrm{ml})$. Cell staining and sorting were performed in PBS with $1 \%$ FCS and $0.1 \%$ azide.

Oligonucleotides. The oligonucleotide sequence containing CpG was TCCATGACGTTCCTGACGTT, and the oligonucleotide control that did not contain CPG motifs (control oligonucleotide, or ODN-CTR) was TCCAGGACTTTCCTCAGGTT. Oligonucleotides were phosphorothioate stabilized and synthesized by Oligos Etc. Inc. (Wilsonville, Oregon, USA). All oligonucleotides were reconstituted in sterile pyrogen-free water and then diluted in PBS for in vivo injections.

Tumor cell lines. The B16 $\left(\mathrm{H}-2^{\mathrm{b}}\right)$ tumor line used is an OVA-transfected clone derived from the murine B16 melanoma (21). B16 tumor cells were cultured in vitro in CM in the presence of geneticin $(2 \mathrm{mg} / \mathrm{ml})$ and hygromycin B $(60 \mu \mathrm{g} / \mathrm{ml})$. The murine colon adenocarcinoma CT26 tumor cell line $\left(\mathrm{H}-2^{\mathrm{d}}\right)$ was purchased from American Type Culture Collection (Rockville, Maryland, USA) and maintained in vitro in CM.

Transduction of tumor cells with CCL20 cDNA. The cDNA encoding CCL20 was obtained by RT-PCR from inflamed mouse skin and cloned into the $\mathrm{P} 3 \mathrm{HN}$ retroviral vector, which contains a CMV promoter. CT26 and B16 parental cell lines were transduced by retroviral infection with a CCL20 cDNA-containing or an empty (mock) vector.

Tumor models. $2 \times 10^{6} \mathrm{CT} 26$ or $5 \times 10^{4} \mathrm{~B} 16$ parental and transduced tumor cells were resuspended in $0.1 \mathrm{ml}$ of PBS and inoculated subcutaneously in the flanks of mice. CpG or ODN-CTR $(80 \mu \mathrm{g}$ each) was resuspended in $30 \mu \mathrm{l}$ of PBS and injected into B16 tumors at day 11 and 14 after tumor inoculation. Where indicated, five CpG injections were delivered every 4 days into the tumors beginning day 11 after tumor challenge. Where indicated, mice received daily intraperitoneal injections of Flt3L $(6 \mu \mathrm{g})$ for 8 consecutive days beginning with the first day of the tumor inoculation. To explore whether the treatment had induced an immune response against distant preestablished nontransduced parental tumor cells, mice were inoculated with $2 \times 10^{6}$ CT 26 or $5 \times 10^{4}$ B16 parental tumors and challenged in the opposite flank 5 days later with CCL20-transduced tumors followed by, in the case of B16 tumors, intratumoral $\mathrm{CpG}$ injections. In some cases BALB/c mice challenged with $2 \times 10^{6}$ CT2 6 parental cells were injected intratumorally daily with $0.1 \mu \mathrm{g}$ of recombinant murine CCL20 protein (PeproTech Inc.) for 3 weeks beginning 1 day after the tumor challenge. Mice were examined twice a week for the presence of tumors. Similarly, C57BL/ 6 mice inoculated with $5 \times 10^{4}$ parental B16 tumor cells were injected intratumorally every day for 3 weeks with $0.1 \mu \mathrm{g}$ of recombinant CCL20 protein alone or in addition to five intratumoral injections of $\mathrm{CPG}$ delivered every 3 days into the tumors beginning day 7 after tumor inoculation. Tumor size represents the product of two perpendicular diameters. Per the protocol, mice were sacrificed when tumors reached $20 \mathrm{~mm}$ in their largest dimension or when ulceration and/or bleeding developed.

Immunobistochemistry. Cryostat-cut tumor sections $(6 \mu \mathrm{m})$ were mounted on slides, air-dried, and stored desiccated at $-80^{\circ} \mathrm{C}$. Immediately before use, sections were fixed for 20 minutes in cold acetone, air-dried for 1 hour, and hydrated in PBS. Tumor sections were incubated with anti-CD16/32 mAb (2.4G2; PharMingen, San Diego, California, USA) for 30 minutes and then stained overnight at $4^{\circ} \mathrm{C}$ with 1:50 dilution of hamster anti-mouse CD11c Ab (HL3; PharMingen) or an isotype control hamster anti-mouse IgG (Ha4/8; PharmMingen) followed by a biotinylated goat anti-hamster IgG (Vector Laboratories Inc., Burlingame, California, USA) for 30 minutes at room temperature. Biotinylated reagents were detected with avidin-alkaline phosphatase (Vector Laboratories Inc.). Enzyme reactions were developed with conventional substrate for alkaline phosphatase (Vector red; Vector Laboratories Inc.). Endogenous alkaline phosphatase activity was blocked with levamisole (Vector Laboratories Inc.). Sections were counterstained with hematoxylin (Vector Laboratories Inc.) and mounted with mounting media (Vector Laboratories Inc.). Images were viewed with a red-filter microscope (DMIRB; Leica Microsystems, Wetzlar, Germany) and processed with Adobe Photoshop software (Adobe Systems Inc., Mountain View, California, USA).

Generation of bone marrow-derived DCs, isolation of tumor cells, and tumor-draining LNs. Bone marrow-DCs (BM-DCs) were generated in the presence of GM-CSF and IL-4 for 6 days as described previously (13). Tumors were surgically removed when they reached a size of 0.5 $\mathrm{cm}$ in diameter, which took 10-15 days. Tumors and LNs were minced into small fragments and incubated in collagenase D (1 $\mathrm{mg} / \mathrm{ml}$; Roche Molecular Biochemicals, Mannheim, Germany) for 30 minutes at $37^{\circ} \mathrm{C}$. Tumor and $\mathrm{LN}$ cell suspensions were blocked with anti-CD16/32 Ab on ice for 10 minutes to minimize nonspecific binding. Anti-mouse mAb to IA-b (AF6-120.1), IA-d (39-10-8), B220 (RA3-6B2), CD3E (145-2C11), CD11c (HL3), CD86 (GL1), CD45 (30-F11), and the second-step Ab (peridinin chloropyll protein-conjugated streptavidin) were purchased from PharMingen.

Effect of the tumor milieu on DC functions. B16 tumors were isolated after 15 days, and tumoral DCs were enriched by magnetic selection using CD11c-conjugated microbeads (Miltenyi Biotec, Auburn, Cal- 
ifornia, USA). Graded numbers of CD $11 c^{+}$-enriched tumoral DCs were irradiated $(30 \mathrm{~Gy})$ and added to $3 \times 10^{5}$ allogeneic $(\mathrm{BALB} / \mathrm{c})$ purified Thy. $1^{+} \mathrm{T}$ cells or Thy. $1^{+}$purified $\mathrm{T}$ cells isolated from the spleen of OVA $257-264-$ specific, MHC class I-restricted, TCR transgenic mice in a final volume of $0.2 \mathrm{ml}$ in 96-well flat-bottom plates (Corning Inc., Corning, New York, USA). Cell proliferation was measured by adding $1 \mu \mathrm{Ci}$ of ${ }^{3} \mathrm{H}$ thymidine per well after 5 days. Irradiated (30 Gy) CD $11 \mathrm{c}^{+}$-enriched tumoral DCs were also cultured in the presence of $5 \times 10^{4} \mathrm{OVA}_{257-264}$-specific, MHC class Irestricted, CD8 $\mathrm{T}$ cell hybridoma (B3Z) for 24 hours at different effector/target $(\mathrm{E} / \mathrm{T})$ ratios. IL-2 release from hybridoma cells was analyzed by ELISA using an IL-2 ELISA kit (R\&D Systems Inc., Minneapolis, Minnesota, USA) following the manufacturer's instructions. Tumor supernatant was collected after 72 hours of culture of $10^{6}$ parental, mock, or CCL20-transduced CT26 or B16 cells in $1 \mathrm{ml}$ RPMI. CD $11 \mathrm{c}^{+}$DCs were enriched from day 6 BM-DC cultures and cultured in $\mathrm{CM}$ or in a $1: 1 \mathrm{mixture}(\mathrm{CM} /$ tumor supernatants), in addition to GM-CSF $(10 \mathrm{ng} / \mathrm{ml})$, for 4 more days. Medium was changed on day 2 . Where indicated, CpG or ODN-CTR $(30 \mu \mathrm{g} / \mathrm{ml})$ was added to the culture.

Effect of B16 milien on BM-DC phenotype. CD $11 c^{+}$DCs were enriched from day 6 BM-DC cultures and cultured in a mixture of $1: 1$ (CM/B16 tumor supernatants) and GM-CSF alone or in addition to $10 \mu \mathrm{g} / \mathrm{ml}$ of neutralizing anti-IL-10 Ab (R\&D Systems Inc.) or 10 $\mu \mathrm{g} / \mathrm{ml}$ of neutralizing anti-TGF- $\beta \mathrm{Ab}(\mathrm{R} \& \mathrm{D}$ Systems Inc.). In some cases CD $11 \mathrm{c}^{+} \mathrm{DCs}$ were cultured in a mixture consisting of $(20 \% \mathrm{CM}$, $40 \%$ B 16 , and $40 \%$ CT26 supernatant) or (20\% CM, 8\% B16, and 72\% CT26 supernatant) and GM-CSF. In all cultures, the medium was changed at day 2 , and expression of costimulatory molecules on gated I-Ab $\mathrm{CD}^{+} \mathrm{CD} 1 \mathrm{c}^{+} \mathrm{DCs}$ was analyzed at day 4 using flow cytometry.

Cytotoxicity assays. Spleen and LNs were isolated from mice at day 30 after the tumor inoculation. Single-cell suspensions were prepared as described above and stimulated with irradiated (100 Gy) parental tumor cells in the presence of $10 \mathrm{U} / \mathrm{ml}$ IL-2. After 5 days of culture, viable effector cells were separated by Lympholyte-M (Cederlane Laboratories Inc., Hornby, Ontario, Canada) gradient centrifugation, and cytotoxic $\mathrm{T}$ cell activity was measured against parental tumor cell targets using a standard 4 -hour ${ }^{51} \mathrm{Cr}$ release assay. Briefly, the target cells were labeled with 100 $\mu \mathrm{Ci}$ of ${ }^{51} \mathrm{Cr}$ for 1 hour, washed, and then incubated for 4 hours with effector cells at different $\mathrm{E} / \mathrm{T}$ ratios in triplicate U-bottom 96-well plates. Spontaneous release and maximum release were determined by incubating target cells without effectors in medium alone or in $10 \%$ SDS, respectively. Spontaneous release was always less than $20 \%$ of maximum. Radioactivity was counted in a liquid scintillation counter and the percentage of specific lysis was measured as the product of [(experi-

Figure 1 mental cpm release - spontaneous cpm release)/(maximal cpm release - spontaneous $\mathrm{cpm}$ release) $] \times 100$.

Lymphocyte depletion. In CT26 tumor experiments, mice were injected intraperitoneally with $0.5 \mathrm{mg}$ of anti-CD8 (2.43), anti-CD4 (GK1.5), or rat control-purified mAb's 1 day before and 1 and 3 days after tumor challenge, and then $0.3 \mathrm{mg}$ Ab's were injected weekly during the course of the experiment. In B16 tumor experiments, CD4 $/-$ or $\mathrm{CD}^{-/-}$mice were used for T cell-dependence experiments. For NK cell depletion, $20 \mu \mathrm{l}$ of rabbit anti-asialo-GM1 serum (Wako Chemicals USA Inc., Richmond, Virginia, USA) or control rabbit serum (Sigma-Aldrich, St. Louis, Missouri, USA) were injected intraperitoneally every 4 days beginning 1 day before the tumor inoculation. Lymphocyte depletion was confirmed in each depletion experiment by FACS analysis of peripheral blood.

Statistical analysis. Differences between groups were analyzed using the Mann-Whitney $U$ test. Survival curves were plotted by the KaplanMeier method. $P$ values less than 0.05 were considered significant.

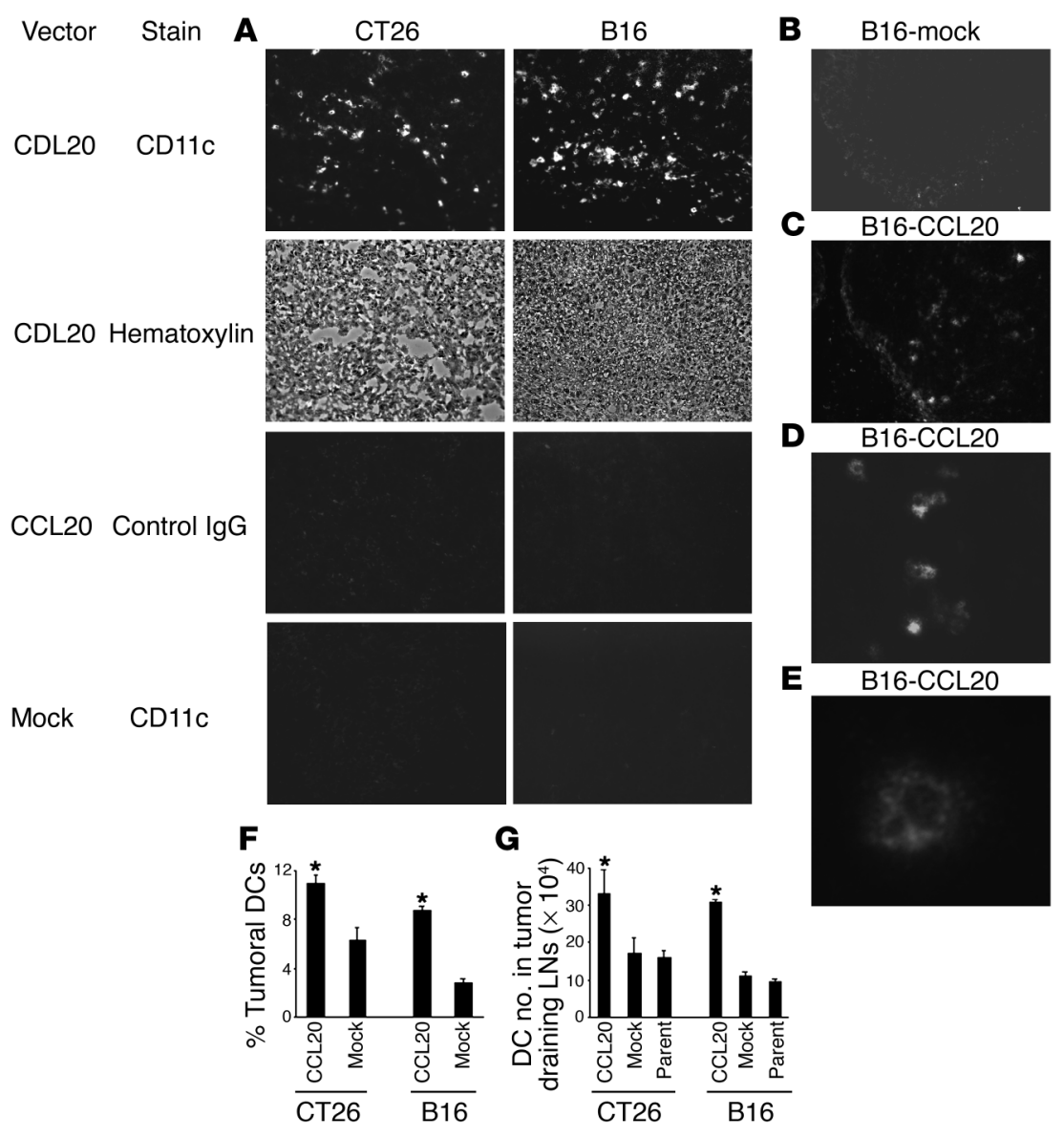

CCL20 expression in the tumor site attracts DCs into and throughout the tumor mass. Mice were inoculated with CT26 or B16 tumors transduced with CCL20 or a mock vector, and DC infiltration at the tumor site was analyzed 10-15 days later. (A) Serial frozen tumor sections were stained with anti-CD11c Ab or isotype IgG control and counterstained with hematoxylin (magnification, $\times 200$ ). Frozen sections of mock-transduced (B) or CCL20-transduced B16 tumors $(\mathbf{C})$ stained with anti-CD11c Ab are shown (magnification, $\times 200$ ). Frozen sections of the core of a CCL20-transduced B16 tumor stained with CD11c Ab are shown at magnifications of $\times 400(\mathbf{D})$ and $\times 600(\mathbf{E})$. Cell suspensions of tumors $(\mathbf{F})$ and tumor-draining LNs (G) were analyzed for the presence of MHC class II+CD11 ${ }^{+}$DCs by flow cytometry. The results shown are the mean of three separate experiments. *Statistical differences between CCL20 and mock experimental groups. 

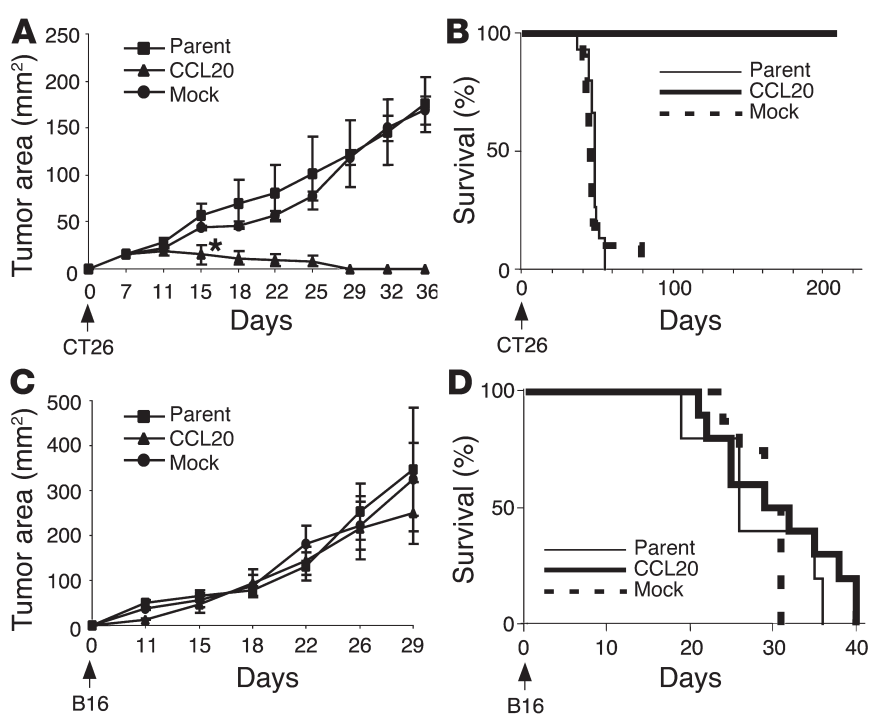

Figure 2

CCL20 expression at the tumor site induces the regression of CT26 but not B16 tumors. (A-D) Graphs showing tumor growth ( $\mathbf{A}$ and $\mathbf{C})$ and survival (B and D) of mice injected with $2 \times 10^{6}$ CT26 (A and B) or $5 \times 10^{4}$ B16 ( $C$ and $\mathbf{D})$ parental tumor cells (parent) or tumor cells transduced with CCL20 or a mock vector. ${ }^{*} P<0.05$ between CCL20 and control groups. In $\mathbf{B}$, survival rate was significantly higher $(P<0.0001)$ in CCL20 versus control groups. The results shown are the mean of five separate experiments in $\mathbf{A}$ and $\mathbf{B}$ and two separate experiments in $\mathbf{C}$ and $\mathbf{D}$.

\section{Results}

CCL20 secretion at the tumor site results in the recruitment of freshly generated blood DCs throughout the tumor. Using a retroviral vector, we transferred the murine CCL20 cDNA into the CT2 6 colon adenocarcinoma and B16 melanoma cell lines. CCL20 expression did not affect the growth of these lines in vitro compared with nontransduced parental cells or parental cells transduced with a mock vector (data not shown). To determine whether CCL20 secreted by tumors was biologically active in vivo, we injected mice subcutaneously with either parental, mock, or CCL20-expressing tumors and analyzed DC infiltration in the tumors. This resulted in a twofold to fourfold increase in the percentage of tumoral DCs and the number of DCs in draining LNs in mice inoculated with CCL20-transduced tumor cells compared with mice inoculated with tumors transduced with a mock vector (Figure 1, A-G). Moreover, we found that in mock-transduced tumors, DCs accumulated mainly at the periphery of the tumors (Figure $1 \mathrm{~B}$ ), while DCs recruited to the CCL2 $0^{+}$tumors penetrated deeply into the tumor core (Figure 1, C-E).

The number of tumoral DCs is the main limiting factor in the antitumor response against CT26 but not B16 tumors. To determine whether an increase in tumoral DCs could restore antitumor immunity in tumor-bearing hosts, we monitored the growth of CCL20 transduced or nontransduced B16 or CT26 tumor cells in vivo. CCL20transduced CT26 tumors did not grow beyond $5-7 \mathrm{~mm}$ in size and started to regress 10-12 days after tumor inoculation, finally disappearing in 30 days (Figure 2A). All mice injected with CCL20-transduced CT2 6 tumors survived for at least 200 days (Figure 2B), while none of the mice injected with parental tumor cells or tumor transduced with a mock vector survived more than 55 days. By contrast, despite a significant increase in the number of tumoral DCs, mice injected with CCL20-transduced B16 tumor cells died 4-6 weeks after the tumor injection in a manner indistinguishable from mice challenged with parental B16 tumors (Figure 2, C and D).

DC targeting to the tumor site, but not systemic DC mobilization with Flt3L, leads to therapeutic antitumor immunity against CT26. Flt3L is a growth factor that induces the proliferation of hematopoietic progenitors in the BM leading to a dramatic and relatively selective increase in circulating DCs $(22,23)$. Since intratumoral CCL20 alone induced a strong immune response against CT26 tumors, we wanted to know whether Flt3L-mediated DC mobilization can also lead to an immune response against these tumors. Thus, BALB/c mice were inoculated subcutaneously with either CCL20 or mocktransduced CT26 tumor cells followed by eight daily intraperitoneal injections of Flt3L beginning with the first day of the tumor challenge. Systemic Flt3L treatment had no effect on tumor growth and did not prolong the survival of the mice (Figure 3, A and B). To learn whether the lack of tumor response observed in Flt3L-treated mice was due to insufficient DC recruitment to the tumor site, we compared the number of tumor-infiltrating DCs in CCL20 and Flt3Ltreated mice. In the Flt3L-treated group the number of DCs infiltrating CT26 tumors was determined 1 day after completion of the Flt3L treatment, which represents the peak of Flt3L-induced DC mobilization (23). The results show that eight daily injections of Flt3L did not lead to substantial DC recruitment into subcutaneous CT26 tumors (Figure 3C) despite a 20- to 50-fold increase of I-Ab+ CD $11 c^{+}$DCs in peripheral LNs and spleen (data not shown).

Activation of tumoral DCs is required for the generation of an antitumor response against $B 16$ tumors. Although CCL20 induced a dramatic influx of DCs throughout B16 tumors (Figure 1), this did not lead to an effective antitumor response, suggesting that the number of
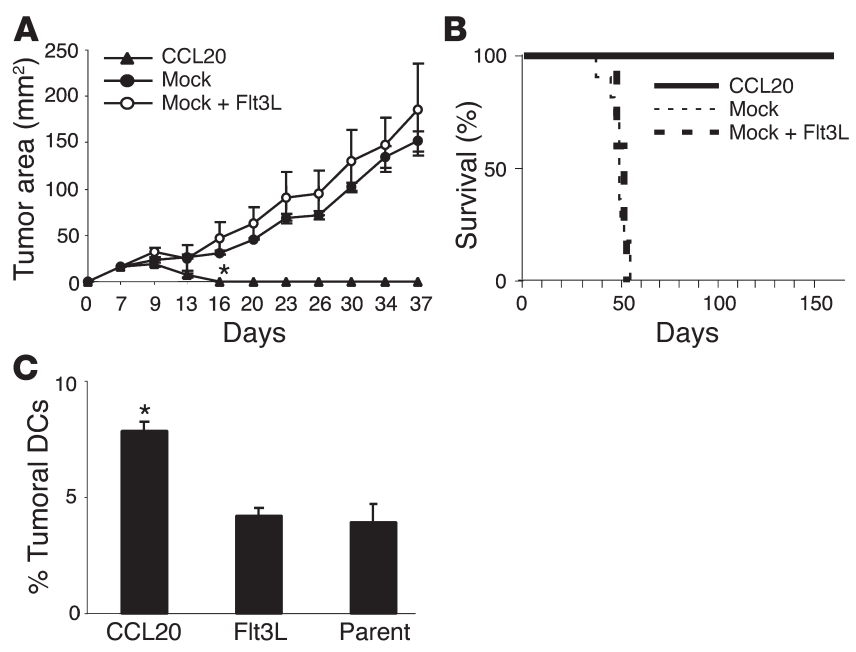

\section{Figure 3}

Systemic Flt3L treatment fails to induce regression of CT26 tumors. (A and B) Graphs show tumor growth and survival of mice inoculated with $2 \times 10^{6}$ CT26 cells transduced with CCL20 or with a mock vector either alone or in addition to eight daily injections of Flt3L, from the first day of the tumor challenge (mock + Flt3L). The results shown are representative of two separate experiments. ${ }^{*} P<0.05$ between CCL20 group and mock, and mock + Flt3L groups. In B, survival rate in CCL20 groups was statistically higher than mock + Flt3L group $(P<0.0001)$. (C) Tumors were isolated 9 days after tumor inoculation (1 day after the last Flt3L injection), and the percentage of DCs relative to total tumor cells was analyzed by flow cytometry. ${ }^{\star} P<0.05$ between CCL20- and Flt3L-treated groups. 
A
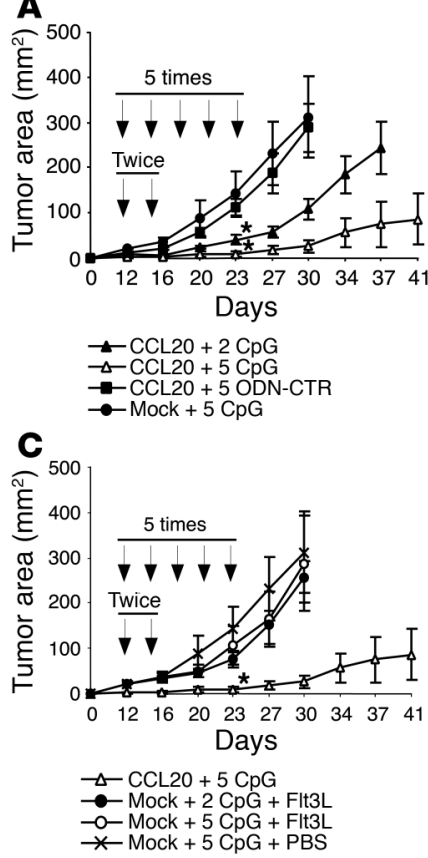

B

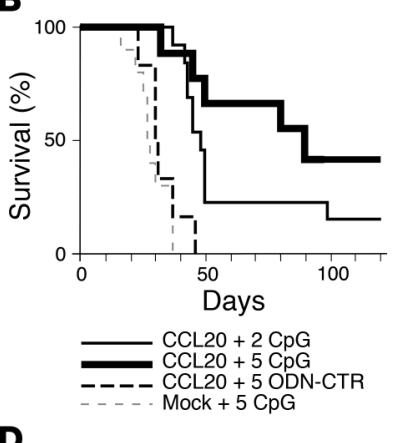

D

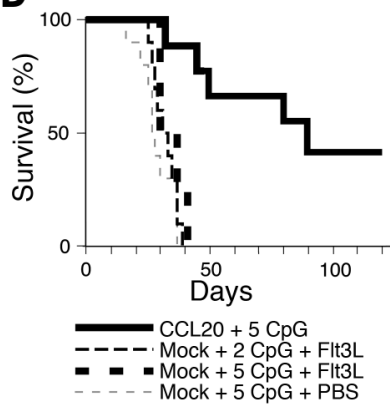

Figure 4

CCL20 + CpG but not Flt3L + CpG induces a therapeutic antitumor response against B16 melanoma. (A and B) Graphs show tumor growth and survival of mice inoculated with $5 \times 10^{4}$ CCL20-transduced B16 tumor cells (CCL20) or mock-transduced tumor cells alone or in addition to two or five intratumoral injections of $\mathrm{CpG}$ or ODN-CTR. Arrows show the days of $\mathrm{CpG}$ injections. (C and D) Mice were inoculated with B16CCL20-transduced tumor cells followed by two or five intratumoral injections of $\mathrm{CpG}$ or with B16-mock-transduced tumor cells followed by two or five intratumoral $\mathrm{CpG}$ injections alone or in addition to eight daily injections of Flt3L beginning on the day of tumor inoculation (mock $+\mathrm{CpG}+$ Flt3L). Results shown are representative of six different experiments in $\mathbf{A}$ and $\mathbf{B}$ and two different experiments in $\mathbf{C}$ and $\mathbf{D}$. (A) $P<0.05$ between $\mathrm{CCL} 20+\mathrm{CpG}$ and control groups. (B) Survival rate was higher in mice treated with CCL20 + 5 CpG compared with mock +5 CpG $(P<0.0001)$ or to CCL20 +5 ODN-CTR $(P=0.0006)$. (C) $P<0.05$ between CCL20 $+\mathrm{CpG}$ and the other groups. (D) Survival rate was significantly higher in mice treated with CCL20 + CpG compared with mock + CpG + Flt3L $(P=0.0012) .{ }^{*} P<0.05$. tumoral DCs was not the only factor limiting anti-B16 immunity. To ask whether modulation of the maturational state of tumoral DCs may restore antitumor immunity against B16, we injected CPG, which is known to induce DC maturation, directly into the tumors at different times after tumor inoculation and monitored tumor growth. We found that intratumoral CPG injections alone did not affect the growth of parental B16 tumors (Figure 4, A and B). By contrast, in mice bearing CCL20-expressing tumors, CpG injections led to a significant antitumor response resulting in a delay of tumor growth $(P<0.05)$. Moreover, survival was significantly prolonged in animals challenged with CCL20-transduced tumor cells and treated with $\mathrm{CpG}$ compared with mice challenged with mock-transduced tumor cells and injected with CPG $(P<0.0001)$ and those challenged with CCL20-transduced tumor cells and treated with ODN-CTR $(P=0.0006$; Figure 4, A and B). Also, additional injections of $\mathrm{CpG}$ into the tumors further improved the survival rate of the mice, although the difference between two and five injections did not reach statistical significance $(P=0.14$; Figure 4 , A and $B)$.

CCL20 plus CPG, but not Flt3L plus CPG, induces therapeutic antitumor immunity against B16 tumors. To determine whether systemically administered Flt3L plus CPG can replace intratumoral CCL20 to induce an effective immune response against B16 tumors, we inoculated mice subcutaneously with B16 mock-transduced tumor cells followed immediately by eight daily intraperitoneal injections of Flt3 $\mathrm{L}$ in addition to two or five CPG tumoral injections and compared tumor growth and animal survival with mice inoculated with CCL20-transduced B16 tumors that were injected with CPG intratumorally. By contrast to CCL20 and CpG, Flt3L and CpG treatment failed to induce any significant antitumor response against $\mathrm{B} 16$ tumors regardless of the number of $\mathrm{CPG}$ injections delivered to the tumors (Figure 4, C and D).

$C p G$ restores the capacity of CCL20-mobilized DCs to present B16 tumor $A g$ 's. At days 15 and 30 after tumor inoculation, mice treated with CCL20-transduced tumors had similar numbers of tumoral DCs, whether or not they received intratumoral injections of CpG (Figure
5A and data not shown). Since DCs were not quantitatively affected by the combined CCL20 and CPG therapy compared with CCL20 alone, we wanted to explore whether the combined therapy affected DC function. Interestingly, CPG injections significantly increased the levels of CD86 and CD80 costimulatory molecules on tumoral DCs and on DCs in tumor-draining LNs (Figure 5, B and C, and data not shown) and increased the capacity of tumoral DCs to stimulate allogeneic T cells (Figure 5D). To determine whether CPG injections could also increase the capacity of CCL20-mobilized tumor DCs to prime a tumor-specific immune response, we cultured freshly isolated tumor-infiltrating DCs with naive and memory OVA-specific T cells. Because B16 tumors were engineered to express OVA, and no exogenous OVA was added to the culture, the only source of OVA Ag available to the tumoral DCs was the tumor cells. Importantly, tumor-infiltrating DCs isolated from CCL20- and CpG-treated tumors, but not DCs isolated from tumors exposed to CCL20 alone, were able to prime naive cells and stimulate memory OVA-specific activated CD8 T cells (Figure 5, E and F). These results suggest that CpG restored the capacity of CCL20-mobilized DCs to cross-present tumor Ag's through the MHC class I pathway, prime Ag-specific naive CD8 T cells, and stimulate Ag-specific effector CD8 T cells.

B16 but not CT26 tumor milieu inhibits the T cell stimulatory capacity of freshly generated BM-derived DCs. Since CCL20-mediated tumoral DC expansion was sufficient to induce an antitumor response against CT2 6 but not B16 tumors, we hypothesized that the B16 but not the CT26 milieu had an inhibitory effect on DCs and that the B16 inhibitory effect can be reversed by CPG. To address this possibility, we cultured ex vivo-generated BM-DCs for 4 days either in medium containing GM-CSF alone or in medium containing GM-CSF and CT26 or B16 tumor supernatant in the presence or absence of CPG. As shown in Figure 6, A and B, B16 supernatant induced downregulation of CD86 costimulatory molecules on BM-DCs and inhibited their capacity to activate allogeneic T cells. Addition of CpG to the medium overcame the inhibitory effect of B16-derived factors on BM-DCs and restored their capacity to activate allogeneic T cells 
A

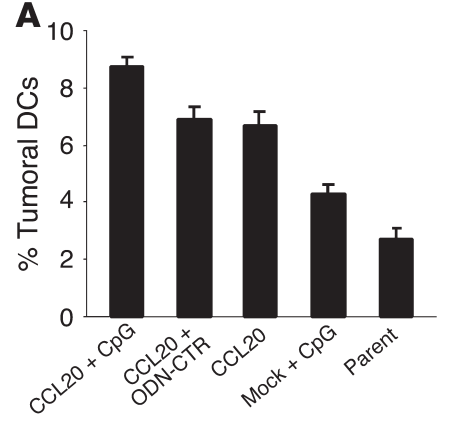

B

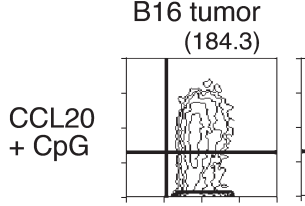

(51.8)

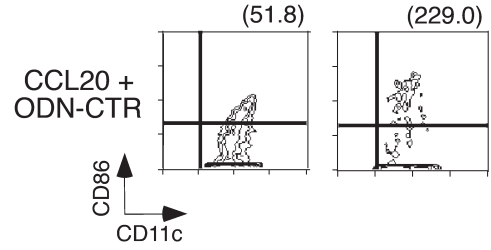

C
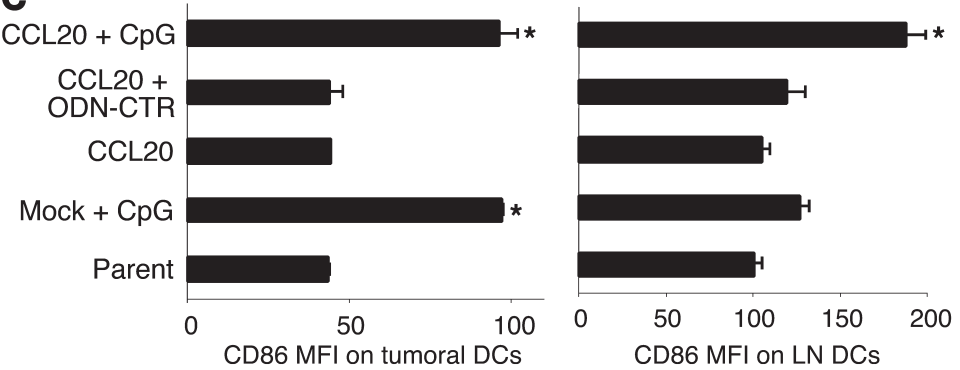
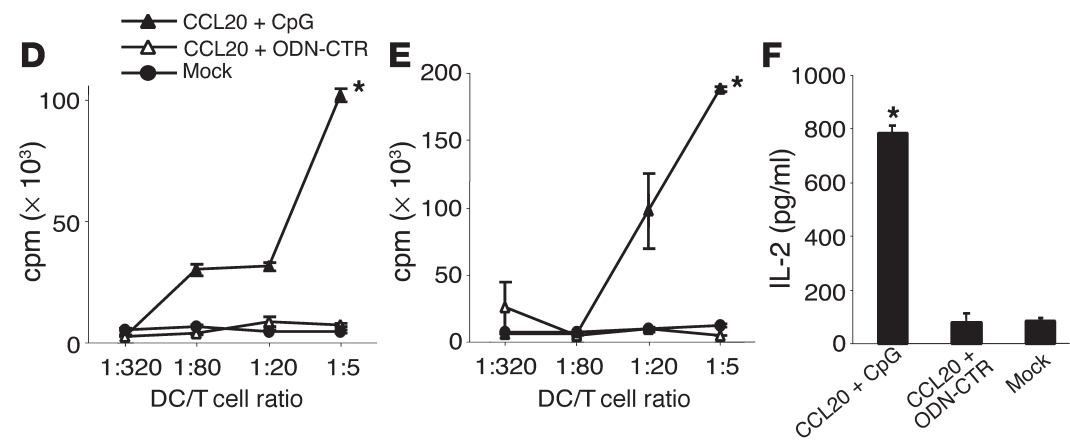

\section{Figure 5}

CpG restores the capacity of B16 tumoral DCs to process tumor Ag's and prime tumor Ag-specific CD8 T cells. Mice were inoculated with B16 parental, B16CCL20, or B16-mock-transduced tumor cells followed by intratumoral injections of $\mathrm{CpG}$ or ODN-CTR. (A) Fifteen days later the percentage of $\mathrm{I}-\mathrm{Ab} \mathrm{b}^{+} \mathrm{CD} 11 \mathrm{c}^{+} \mathrm{DCs}$ present in the tumors was analyzed. (B) Dot plot shows the expression of CD86 on I-Ab+ $\mathrm{CD} 11 \mathrm{c}^{+}$gated DCs. CD86 mean fluorescence intensity (MFI) is shown in parentheses above each quadrant. One representative experiment out of three is shown. (C) Graphs show CD86 MFI on gated tumoral DCs and DCs in tumordraining LNs. Results shown are the mean of three separate experiments. (D-F) Graded numbers of purified $\mathrm{CD} 11 \mathrm{C}^{+}$tumoral $\mathrm{DC}$ s were cultured in the presence of $3 \times 10^{5}$ purified allogeneic BALB/c T cells $(D)$ or $3 \times 10^{5}$ purified CD8 OVA ${ }_{257-264}-\mathrm{TCR}$ transgenic T cells $(\mathrm{E})$, and $T$ cell proliferation was measured 5 days later. Purified $\mathrm{CD} 11 \mathrm{C}^{+}$tumoral $\mathrm{DC}$ s were cultured in the presence of $5 \times 10^{4} \mathrm{OVA}_{257-264}$-specific CD8 T cells (clone B3Z), and IL-2 secretion was measured by ELISA 24 hours later $(\mathbf{F})$. Results shown are representative of two separate experiments. ${ }^{*} P<0.05$ between $\mathrm{CpG}$-injected groups and group controls.
(Figure 6, A and B). By contrast, CT26 tumor supernatants did not alter the function of in vitro-generated BM-DCs (Figure 6, A and B). Similarly, tumor-infiltrating DCs isolated from parental or CCL20transduced CT26 tumors had higher levels of costimulatory molecules (Figure 6C) compared with DCs infiltrating CCL20-transduced B16 tumors (Figure 5, B and C), consistent with the observation that the CT26 tumor milieu does not suppress DC immune functions. These results likely explain the differential antitumor effects induced by CCL20 against B16 and CT26 tumors. Interestingly, B16-mediated downregulation of costimulatory molecules on DCs was partially blocked when CT26 supernatant was added to the cultures, suggesting that CT26 may enhance DC activation in vivo in a manner that favors the development of an immune response to the tumor (Figure 6D). We also found that the inhibitory effect of the B16 milieu on DCs was not blocked by neutralizing Ab's to TGF- $\beta$ and IL-10 cytokines (Figure 6E).

CTLs mediate the antitumor response induced by CCL20 and CPG. In mice inoculated with CCL20-transduced CT2 6 tumors, tumor-specific CTLs were easily detected (Figure 7A), consistent with our finding that the immune functions of DCs present in CT26 tumors were not altered by the tumor environment. We also found that the antitumor response induced by CCL20 alone was dependent on both $\mathrm{CD}^{+}$and $\mathrm{CD}^{+} \mathrm{T}$ cells, but independent of NK cells (Figure 7B). By contrast, in mice inoculated with B16 tumors, tumor-specific CTLs were detected only if CCL20 was expressed at the tumor site and the mice received intratumoral $\mathrm{CPG}$ injections (Figure 7C). Interestingly, the antitumor response induced by CCL20 and CPG was dependent on CD8 cytotoxic T cells but independent of CD4 help (Figure 7D).

Intratumoral CCL2O and CPG induce the regression of established tumors at distant sites. We wanted to explore whether CCL20 expression at an accessible tumor site in the presence or absence of local CPG injections can induce a systemic antitumor response against established tumors in other locations. Thus, mice were first inoculated with parental tumor cells and 5 days later challenged in the contralateral flank with CCL20-transduced CT26 or CCL20-transduced B16 tumor cells, followed or not followed by CPG injections into B16 tumors. Although complete regression of the distant tumors was not obtained, CCL20 alone in the case of CT26 tumors and CCL20 plus CPG in the case of B16 tumors induced a systemic antitumor response against these previously established nontransduced tumors (Figure 8, A and B).

Injection of CCL20 protein into parental tumors can induce a therapeutic antitumor response. Since CCL20 was required to induce an antitumor response in two different tumor models, we wanted to explore alternative and more practical ways of delivering CCL20 into the tumors. Therefore, mice inoculated with CT26 tumors received daily intratumoral injections of CCL20 protein for 3 weeks begin- 

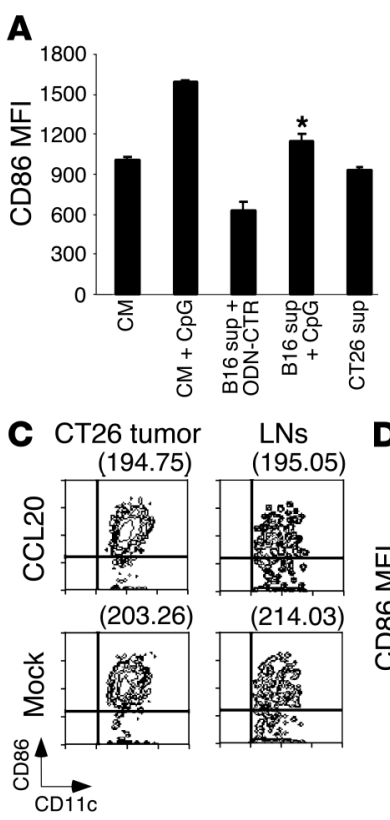

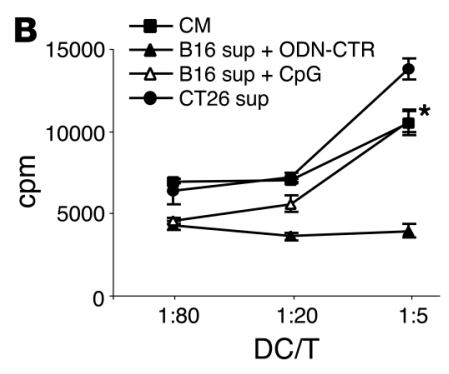

D

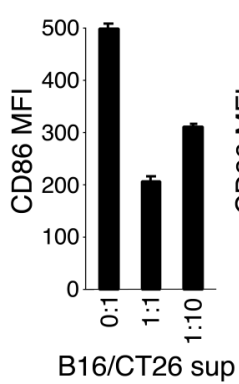

E

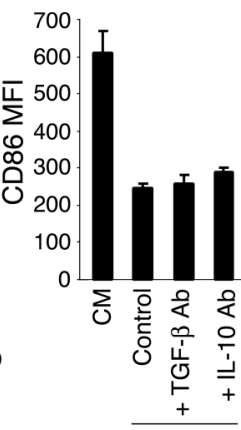

B16 sup

ning 1 day after tumor inoculation. As shown in Figure 8, C and D, such injections induced the regression of parental CT26 tumors. Although the growth of B16 tumors treated with CCL20 and CPG was reduced compared with the control groups, this effect was not statistically significant (Figure 8, E and F).

\section{Discussion}

Using two different tumor models, we have shown that the number and maturation status of tumoral DCs are important limiting factors in DC-based immunity and that DCs can be manipulated directly at the tumor site to induce a systemic antitumor response without the need for tumor Ag vaccination or systemic DC mobilization.

\section{Figure 7}

CCL20 alone or in combination with CpG induces a systemic T cell-dependent antitumor response. Mice were inoculated subcutaneously with $2 \times 10^{6}$ CT26 parent, CT26 mock, or CT26-CCL20-transduced tumor cells (A and B), or $5 \times 10^{4}$ B16 parent, B16 mock, or B16CCL20 tumor cells in addition to intratumoral injections of $\mathrm{CpG}$ or ODN-CTR ( $\mathbf{C}$ and $\mathbf{D}$ ). Thirty days later graded numbers of spleen and LN cells were cultured in the presence of irradiated parental tumors for 5 days, and tumor-specific cytotoxic $T$ cell activity against nontransduced parental tumor cell targets was measured using a standard $4-h o u r{ }^{51} \mathrm{Cr}$ release assay as described in Methods ( $\mathbf{A}$ and $\mathbf{C}$ ). Results shown are representative of two separate experiments. (B) Mice were injected intraperitoneally with depleting anti-CD8, anti-CD4, or control rat Ab's to deplete circulating T cells, or anti-asialo-GM1 serum, or control rabbit serum for NK cell depletion. ${ }^{*} P<0.05$ between CCL20/control IgG and CCL20/CD4 Ab- and CCL20/CD8 Ab-treated groups. (D) CD4--, CD8 ${ }^{-1}$, or WT mice were inoculated with B16-CCL20 tumor cells followed by intratumoral $\mathrm{CpG}$ injections. NK cell depletion was done as in $\mathbf{B} .{ }^{*} P<0.05$ between WT mice and CD8 ${ }^{-1-}$ mice treated with CCL20 + CpG. There was no significant difference in tumor growth between WT mice and $\mathrm{CD}^{-1-}$ mice treated with CCL20 + CpG.

\section{Figure 6}

B16- but not CT26 tumor-derived factors inhibit the immunostimulatory capacity of freshly generated BM-DCs. (A and B) CD11 $\mathrm{c}^{+}$-enriched DCs from BM-DCs cultures were incubated for 4 days in $C M$ or in the presence of CT26 supernatant (CT26 sup) or B16 supernatant (B16 sup) in addition to CpG or ODN-CTR. (A) Graphs show the MFI of CD86 costimulatory molecules on gated IA-b+ CD11 $c^{+}$DCs in different culture conditions. (B) Graded numbers of CD11 $\mathrm{c}^{+}$-enriched BM-DCs cultured for 4 days in different culture conditions were added to $3 \times 10^{5}$ allogeneic (BALB/c) spleen T cells, and T cell proliferation was measured 5 days later. Results shown are representative of three different experiments. ${ }^{*} P<0.05$ between B16 supernatant + CpG group compared with B16 supernatant + ODN group. (C) Mice were inoculated with CT26-CCL20 or CT26-mock-transduced tumor cells, and 10 days later CD11 $\mathrm{c}^{+}$tumoral DCs were analyzed by flow cytometry. CD86 MFI is written in parentheses above each quadrant. (D and E) Graphs show the MFI of CD86 costimulatory molecules on gated IA-b+ CD11 $c^{+}$DCs cultured under different conditions. (D) CD11 $\mathrm{C}^{+}$-enriched DCs were incubated in B16 supernatant alone or in a 1:1 or 1:10 mixture with CT26 supernatant (B16/CT26 sup) as described in Methods. (E) CD11 $\mathrm{c}^{+}$-enriched DCs were incubated in CM or in B16 supernatant alone or in addition to neutralizing $\mathrm{Ab}$ to TGF- $\beta$ or IL-10.

Targeted recruitment of DCs to the tumor site was achieved by CCL20 chemokine. CCL20 is a C-C chemokine expressed by most inflamed tissues (2), but it is also constitutively secreted in some tissues, including the lung and liver (24). The only known receptor for CCL20 is CCR6 $(25,26)$, a molecule expressed by numerous leukocytes, including DCs (27), T cells (28), mature peripheral B cells (29-31), and a subset of germinal center B cells (29), while it is absent on monocytes and neutrophils (30). Among DCs, CCR6 is expressed at high levels by lung DCs, most CD $11 \mathrm{~b}^{+}$DCs in the spleen (30), Peyer's patches (32), epidermal Langerhans cells (LCs) (30s), and CD $34^{+}$-derived LCs (27), but not by monocyte-derived DCs (27), suggesting that CCR6 expression may contribute to the

A
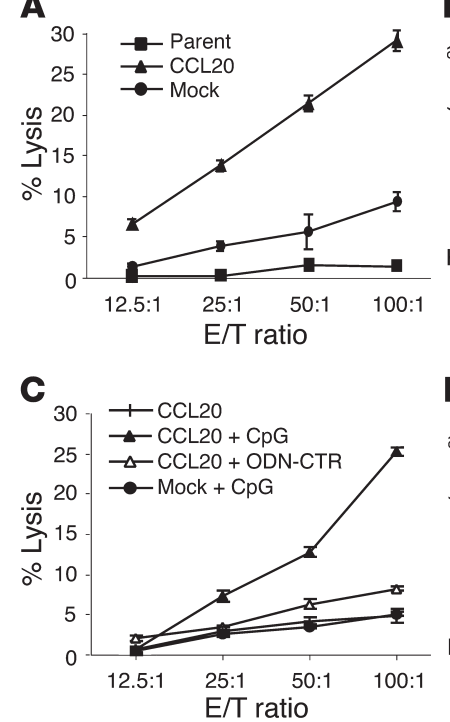

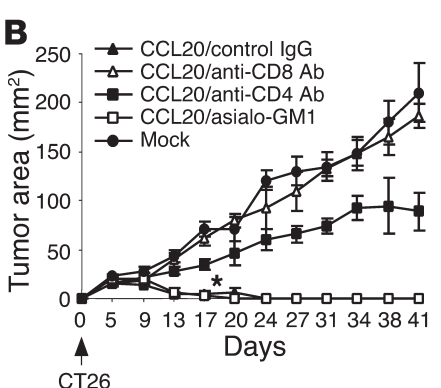

СТ26

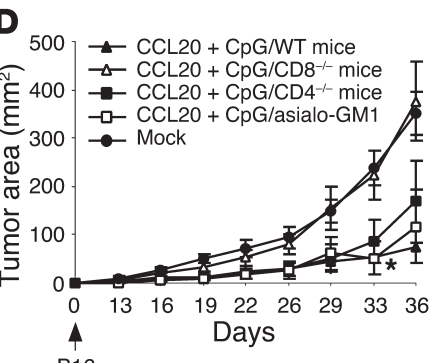



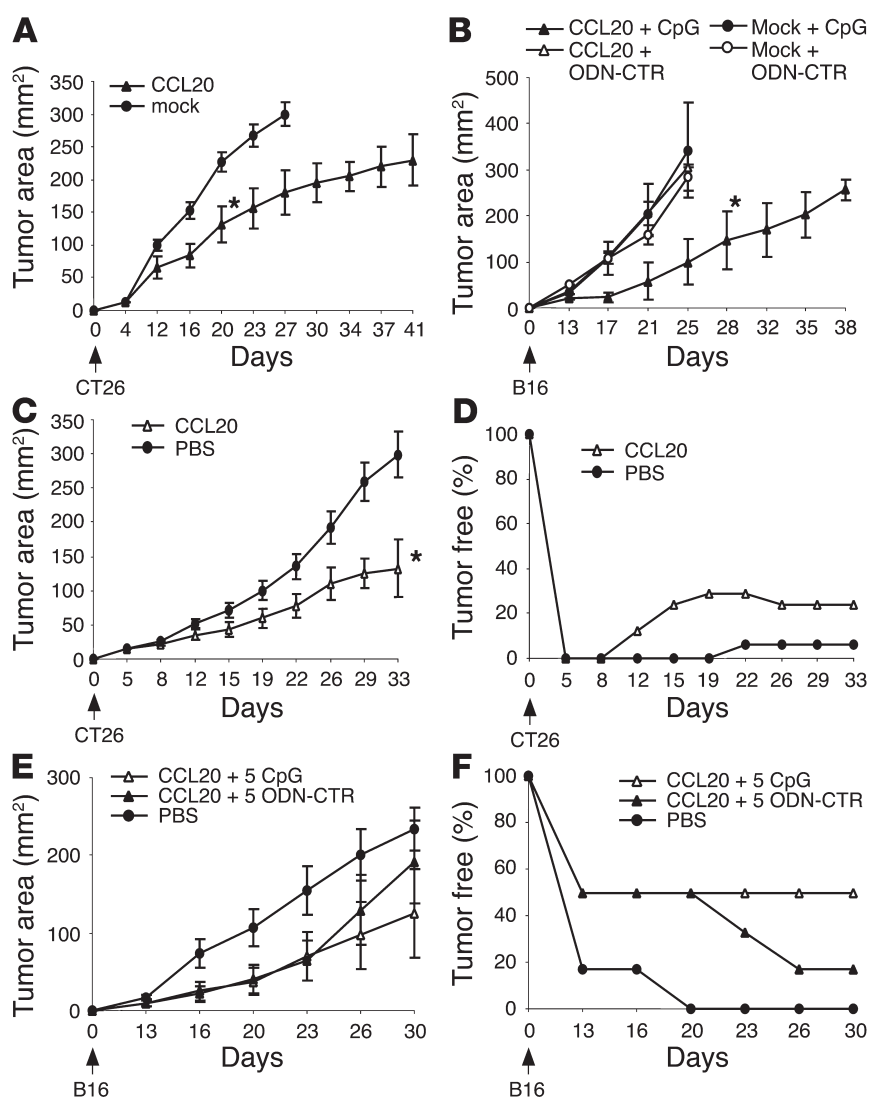

Figure 8

Intratumoral CCL20 alone or in combination with CpG induces the regression of preexisting parental tumors. (A and $\mathbf{B})$ Mice were inoculated subcutaneously with $2 \times 10^{6}$ CT26 parental $(\mathbf{A})$ or $5 \times 10^{4}$ B16 parental tumor cells (B). Five days later the mice were injected in the opposite flank with CT26 mock or CT26-CCL20-transduced tumor cells (A) or B16 mock or B16-CCL20-transduced tumor cells in addition to two intratumoral CpG or ODN-CTR injections (B). Graphs show the growth of distant parental tumors. Results shown are representative of two separate experiments. (A) $P<0.05$ between CCL20 and mock groups; (B) $P<0.05$ between CCL20 + CpG and the other groups. (C and $\mathbf{D})$ Mice were inoculated with $2 \times 10^{6} \mathrm{CT} 26$ parental tumors, and $0.1 \mu \mathrm{g}$ of recombinant CCL20 protein or PBS was injected daily for 3 weeks into the tumors beginning 1 day after tumor inoculation. Results shown are from three separate experiments. * Statistical difference in tumor growth between mice treated with CCL20 compared with untreated mice $(P<0.05)$. (E and F) Mice were inoculated with $5 \times 10^{4}$ B16 parental tumors, and recombinant CCL20 protein was injected into the tumors alone or in addition to five intratumoral injections of $\mathrm{CpG}$ as described in Methods.

tissue selectivity of DC recruitment. Importantly, CCR6 is present mainly on immature DCs and is downregulated during DC maturation. CCR6 expression on immature DCs allows their migration toward a gradient of CCL20 chemokine expressed in most inflamed tissues where they will take up and process Ag's before migrating to the draining LNs. In our study, CCL20 was expressed at the tumor site either by CCL20 gene transduction of tumor cells or by intratumoral injections of CCL20 recombinant protein. In both cases CCL20 was biologically active in vivo and able to attract DCs into the tumor core, suggesting that the tumor milieu does not prevent the diffusion and action of the chemokine gradient. The ability of CCL20 to attract DCs into and throughout the tumors is impor- tant, since in most studied cancer patients, DCs have been found mainly at the periphery of the tumors $(7,10)$, which may limit their interaction with tumors cells. Importantly, the capacity of CCL20 to recruit circulating DCs to the tumor site exposes tumor cells to freshly generated DCs (as opposed to local DCs), which may be less affected by an immunosuppressive tumor milieu. It is interesting to note that tumor growth was significantly reduced in mice treated with low doses of recombinant CCL20 protein compared with untreated mice or mice treated with higher doses of CCL20 (data not shown), underlining the fact that chemoattraction of leukocytes is a tightly regulated process.

CCL20 expression at the tumor site was sufficient to induce activation of a specific antitumor CD4 and CD8 T cell response against parental CT26 tumors, suggesting that DCs present in these tumors were not inhibited by the tumor milieu. This was confirmed by an experiment showing that DCs freshly isolated from CT26 tumors were mature and that the CT26 supernatant did not inhibit the capacity of freshly generated BM-DCs to activate allogeneic T cells. These results suggest that the low number of tumoral DCs is the main limiting factor in DC-mediated antitumor immunity against CT26 tumors. The absence of inhibition of BM-DC by factors present in the CT26 tumor milieu is in contrast with a recent report showing that CT26 tumors contain mostly immature DCs and inhibit the capacity of BM-DCs to secrete cytokines in response to innate stimuli (8). These differences are probably the result of acquired differences that may affect the immunogenicity of one transformed cell line maintained in separate laboratories and underlines the variability among tumors even of the same origin.

By contrast to CT26 tumors, CCL20 expression in B16 tumors was not sufficient to induce an antitumor response despite a clear increase of tumor-infiltrating DCs. CPG, in addition to CCL20, was always required to induce a strong systemic antitumor immune response against B16 tumors. This contrasts with a previous study showing that intratumoral expression of CCL20 alone can lead to the regression of B16 tumors (33). In the latter study, however, the CCL20 gene was delivered to the tumors by an adenovirus vector that is known to induce DC maturation and activation (33). The requirement in our study for $\mathrm{CpG}$ injections for the induction of an antitumor response against B16 may be due to the fact that B16 tumors, in contrast to CT26 tumors, can inhibit the stimulatory capacity of tumoral DCs as suggested by the inhibitory effect of B16 tumor supernatants on freshly cultured BM-DCs. Because CPG fails to costimulate $T$ cells activated with anti-CD3 or anti-TCR, but enhances the capacity of APCs to activate T cells $(34,35)$, it is likely that the CCL20 and CPG antitumor effect was not due to a direct effect on $\mathrm{T}$ cells but rather on APCs. Indeed, we found that CpG could reverse the inhibitory effect of the B16 tumor milieu on the expression of costimulatory molecules and on the capacity of purified BM-DCs and tumoral DCs to stimulate allogeneic T cells. Moreover, CpG-treated, CCL20-mobilized DCs were able to crosspresent exogenous tumor Ag's through the MHC class I pathway and prime naive and effector tumor Ag-specific CD8 T cells ex vivo. Similarly, the addition of CpG to CCL20 led to a CD8-dependent therapeutic antitumor response, which was independent of CD4 help. This is consistent with a study showing that $\mathrm{CpG}$ can induce DC cross-presentation of exogenous Ag's through the class I pathway (36) as well as findings from our group and others that a vaccine combining $\mathrm{CpG}$ and Ag induces similar levels of Ag-specific CD8 cytotoxic T cells (37) and tumor protection in CD4 WT and CD4-deficient mice $(13,38)$. 
A recent study has explored the capacity of $\mathrm{CPG}$ to activate tumoral DCs and restore their capacity to induce an immune response. In this study, coadministration of anti-IL-10 receptor $\mathrm{Ab}$ to $\mathrm{CPG}$ injections was necessary to obtain an antitumor response (8). In our model, CCL20 and CpG were sufficient to induce strong and durable immunity even in the absence of anti-IL-10 receptor $\mathrm{Ab}$. It is thus possible that the presence of a higher number of freshly recruited DCs from the circulation can eliminate the need for anti-IL-10, although it will be interesting to explore whether addition of anti-IL-10 Ab enhances the antitumor effect of CCL20 and $\mathrm{CpG}$ therapy. We also found that neutralizing Ab's to IL-10 or TGF- $\beta$, cytokines known to inhibit the ability of DCs to initiate a $\mathrm{T}$ cell immune response $(39,40)$, failed to reverse the inhibitory effect mediated by the B16 milieu on DCs in vitro, suggesting that these cytokines may not play an important role in our model. Repeated high doses of peritumoral injections of CpG could also lead to tumor rejection in B16 models $(41,42)$. We found, however, that the combination of CCL20 and CpG was far more effective than $\mathrm{CpG}$ alone, underlining the importance of expanding the number of DCs at the tumor site.

In addition to their well-documented role in attracting leukocytes to specific sites, chemokines have other effects that may enhance an antitumor response. For example, CCL21 has been shown to inhibit tumor angiogenesis as well as increase DC recruitment to tumor sites and enhance the CD8 antitumor response (43). On the other hand CCL2/MCP-1 chemokine, which can attract different DC subsets and monocytes, was shown to promote tumor angiogenesis through its action on endothelial cells and thus promote tumor growth (44). In our study, the antitumor effect mediated by CCL20 and $\mathrm{CpG}$ was dependent mainly on the induction of a specific antitumor CD8 T cell response since depletion of CD8 T cells abrogated the therapeutic effect induced by CCL20 alone in the case of CT26 and by CCL 20 plus CPG in the case of B16 tumors. Importantly, the observation that CCL20 alone and the combined CCL20 plus CPG treatment were able to induce a clinical response against distant parental CT26 and B16 tumors, respectively, suggests the induction of systemic immunity that may be able to induce the regression of metastatic tumors.

Flt $3 \mathrm{~L}$ is a growth factor that induces the proliferation of DC progenitors in BM, leading to a 20- to 80-fold increase of DCs in lymphoid organs (23). Flt3L alone has been reported to induce the regression of chemically induced sarcomas in mice (45), but this result was not reproduced by others $(46,47)$. Here we show that systemic injections of Flt3 $\mathrm{L}$ in mice induced a much lower recruitment of DCs to the tumor site compared with intratumoral expression of CCL20. The low number of tumoral DCs, in contrast to the high number of DCs found in lymphoid organs after Flt3L injections, suggests that Flt3L-mobilized DCs home preferentially to lymphoid tissues and have a poor capacity to home to tumors. We have previously shown that Flt3 L treatment followed by subcutaneous administration of $\mathrm{CpG}$ mixed with a tumor $\mathrm{Ag}$ can induce a therapeutic immune response against B16 tumors (13). In this published study, immunization was done in the mouse footpad contralateral to the site where tumor cells were injected, and a strong CD8 antitumor response was generated in the draining LNs. There was no tumor response in the absence of tumor Ag vaccination, however. In the current study we found that systemic administration of Flt $3 \mathrm{~L}$ followed by intratumoral CpG injections failed to induce an antitumor response. This was probably due to the fact that Flt3L did not attract a sufficient number of DCs to the tumor site despite dramatic increases of DCs in the circulation and in lymphoid organs and suggests that a threshold number of intratumoral DCs must be reached to induce antitumor immunity.

Our results demonstrate the importance of expansion and activation of DCs in the tumor core for the induction of effective antitumor immunity. For tumors that do not inhibit DC function, an increase in tumoral DCs may be sufficient to induce antitumor immunity, while for immunosupressive tumors, both expansion and activation of tumoral DCs are required. These results also emphasize the importance of the tumor environment for the design of cancer vaccines and provide the basis for the use of chemokine-based strategies for clinical studies in cancer patients. The ability to deliver CCL20 protein alone or in combination with CpG offers new possibilities for the treatment of patients with accessible tumors.

\section{Acknowledgments}

We thank Motoko Furumoto for her help in formatting the manuscript and Claudia Benike for critically reviewing the manuscript. This work was supported by a grant from the National Heart, Lung and Blood Institute (HL57443) and a grant from the State of California (9RT-0229).

Received for publication August 8, 2003, and accepted in revised form December 16, 2003.

Address correspondence to: Edgar G. Engleman, 800 Welch Road, Palo Alto, California 94304, USA. Phone: (650) 723-7960; Fax: (650) 725-0592; E-mail: edengleman@stanford.edu.

Miriam Merad's present address is: Mount Sinai School of Medicine, Institute for Gene Therapy and Molecular Medicine, New York, New York, USA.
1. Banchereau, J., and Steinman, R.M. 1998. Dendritic cells and the control of immunity. Nature. 392:245-252

2. Banchereau, J., et al. 2000. Immunobiology of dendritic cells. Annu. Rev. Immunol. 18:767-811.

3. Gabrilovich, D.I., et al. 1996. Production of vascular endothelial growth factor by human tumors inhibits the functional maturation of dendritic cells. [erratum 1996, 2:1267]. Nat. Med. 2:1096-1103.

4. Gabrilovich, D.I., Corak, J., Ciernik, I.F., Kavanaugh, D., and Carbone, D.P. 1997. Decreased antigen presentation by dendritic cells in patients with breast cancer. Clin. Cancer Res. 3:483-490.

5. Menetrier-Caux, C., et al. 1998. Inhibition of the differentiation of dendritic cells from CD34(+) progenitors by tumor cells: role of interleukin- 6 and macrophage colony-stimulating factor. Blood. 92:4778-4791.
6. Nestle, F.O., Burg, G., Fah, J., Wrone-Smith, T., and Nickoloff, B.J. 1997. Human sunlight-induced basalcell-carcinoma-associated dendritic cells are deficient in $\mathrm{T}$ cell co-stimulatory molecules and are impaired as antigen-presenting cells. Am. J. Pathol. 150:641-651.

7. Bell, D., et al. 1999. In breast carcinoma tissue, immature dendritic cells reside within the tumor, whereas mature dendritic cells are located in peritumoral areas. J. Exp. Med. 190:1417-1426.

8. Vicari, A.P., et al. 2002. Reversal of tumor-induced dendritic cell paralysis by CPG immunostimulatory oligonucleotide and anti-interleukin 10 receptor antibody. J. Exp. Med. 196:541-549.

9. Vicari, A.P., Caux, C., and Trinchieri, G. 2002. Tumour escape from immune surveillance through dendritic cell inactivation. Semin. Cancer Biol. 12:33-42.
10. Vermi, W., et al. 2003. Recruitment of immature plasmacytoid dendritic cells (plasmacytoid monocytes) and myeloid dendritic cells in primary cutaneous melanomas. J. Pathol. 200:255-268.

11. Fong, L., and Engleman, E.G. 2000. Dendritic cells in cancer immunotherapy. Annu. Rev. Immunol. 18:245-273.

12. Dhodapkar, M.V., Steinman, R.M., Krasovsky, J. Munz, C., and Bhardwaj, N. 2001. Antigen-specific inhibition of effector $\mathrm{T}$ cell function in humans after injection of immature dendritic cells. J. Exp. Med. 193:233-238.

13. Merad, M., Sugie, T., Engleman, E.G., and Fong, L. 2002. In vivo manipulation of dendritic cells to induce therapeutic immunity. Blood. 99:1676-1682.

14. Cui, Y., et al. 2003. Immunotherapy of established tumors using bone marrow transplantation with 
antigen gene-modified hematopoietic stem cells. Nat. Med. 9:952-958.

15. Caux, C., et al. 2000. Dendritic cell biology and regulation of dendritic cell trafficking by chemokines. Springer Semin. Immunopathol. 22:345-369.

16. Cyster, J.G. 1999. Chemokines and cell migration in secondary lymphoid organs. Science. 286:2098-2102.

17. Cyster, J.G. 1999. Chemokines and the homing of dendritic cells to the $T$ cell areas of lymphoid organs. J. Exp. Med. 189:447-450.

18. Hogquist, K.A., et al. 1994. T cell receptor antagonist peptides induce positive selection. Cell. 76:17-27.

19. Rahemtulla, A., et al. 1991. Normal development and function of CD8+ cells but markedly decreased helper cell activity in mice lacking CD4. Nature. 353:180-184

20. Fung-Leung, W.P., et al. 1991. CD8 is needed for development of cytotoxic $\mathrm{T}$ cells but not helper $\mathrm{T}$ cells. Cell. 65:443-449.

21. Mayordomo, J.I., et al. 1995. Bone marrow-derived dendritic cells pulsed with synthetic tumour peptides elicit protective and therapeutic antitumour immunity. Nat. Med. 1:1297-1302.

22. McKenna, H.J., de Vries, P., Brasel, K., Lyman, S.D., and Williams, D.E. 1995. Effect of flt 3 ligand on the ex vivo expansion of human CD34+ hematopoietic progenitor cells. Blood. 86:3413-3420.

23. Maraskovsky, E., et al. 1996. Dramatic increase in the numbers of functionally mature dendritic cells in Flt3 ligand-treated mice: multiple dendritic cell subpopulations identified. J. Exp. Med. 184:1953-1962.

24. Hieshima, K., et al. 1997. Molecular cloning of a novel human CC chemokine liver and activation-regulated chemokine (LARC) expressed in liver. Chemotactic activity for lymphocytes and gene localization on chromosome 2. J. Biol. Chem. 272:5846-5853.

25. Baba, M., et al. 1997. Identification of CCR6, the specific receptor for a novel lymphocyte-directed CC chemokine LARC. J. Biol. Chem. 272:14893-14898.

26. Varona, R., et al. 1998. Molecular cloning, functional characterization and mRNA expression analysis of the murine chemokine receptor CCR6 and its specific ligand. MIP-3alpha. FEBS Lett. 440:188-194.
27. Dieu, M.C., et al. 1998. Selective recruitment of immature and mature dendritic cells by distinct chemokines expressed in different anatomic sites. J. Exp. Med. 188:373-386.

28. Liao, F., et al. 1999. CC-chemokine receptor 6 is expressed on diverse memory subsets of T cells and determines responsiveness to macrophage inflammatory protein 3 alpha. J. Immunol. 162:186-194.

29. Bowman, E.P., et al. 2000. Developmental switches in chemokine response profiles during B cell differentiation and maturation. J. Exp. Med. 191:1303-1318.

30. Kucharzik, T., Hudsafon, I.J., Waikel, R.L., Martin, W.D., and Williams, I.R. 2002. CCR6 expression distinguishes mouse myeloid and lymphoid dendritic cell subsets: demonstration using a CCR6 EGFP knock-in mouse. Eur. J. Immunol. 32:104-112.

31. Liao, F., Shirakawa, A.K., Foley, J.F., Rabin, R.L., and Farber, J.M. 2002. Human B cells become highly responsive to macrophage-inflammatory protein-3 alpha/CC chemokine ligand-20 after cellular activation without changes in CCR6 expression or ligand binding. J. Immunol. 168:4871-4880.

32. Iwasaki, A., and Kelsall, B.L. 2000. Localization of distinct Peyer's patch dendritic cell subsets and their recruitment by chemokines macrophage inflammatory protein (MIP)-3alpha, MIP-3beta, and secondary lymphoid organ chemokine. J. Exp. Med. 191:1381-1394.

33. Fushimi, T., Kojima, A., Moore, M.A., and Crystal, R.G. 2000. Macrophage inflammatory protein 3alpha transgene attracts dendritic cells to established murine tumors and suppresses tumor growth. J. Clin. Invest. 105:1383-1393.

34. Wagner, H. 1999. Bacterial CpG DNA activates immune cells to signal infectious danger. Adv. Immunol. 73:329-368.

35. Kranzer, K., et al. 2000. CpG-oligodeoxynucleotides enhance T-cell receptor-triggered interferon-gamma production and up-regulation of CD69 via induction of antigen-presenting cell-derived interferon type I and interleukin-12. Immunology. 99:170-178.

36. Datta, S.K., et al. 2003. A subset of Toll-like receptor ligands induces cross-presentation by bone marrow- derived dendritic cells. J. Immunol. 170:4102-4110

37. Sparwasser, T., Vabulas, R.M., Villmow, B., Lipford, G.B., and Wagner, H. 2000. Bacterial CpG-DNA activates dendritic cells in vivo: $\mathrm{T}$ helper cell-independent cytotoxic $\mathrm{T}$ cell responses to soluble proteins. Eur. J. Immunol. 30:3591-3597.

38. Cho, H.J., et al. 2000. Immunostimulatory DNAbased vaccines induce cytotoxic lymphocyte activity by a T-helper cell-independent mechanism. Nat. Biotechnol. 18:509-514.

39. Steinbrink, K., Wolfl, M., Jonuleit, H., Knop, J., and Enk, A.H. 1997. Induction of tolerance by IL-10 treated dendritic cells. J. Immunol. 159:4772-4780.

40. Letterio, J.J., and Roberts, A.B. 1998. Regulation of immune responses by TGF-beta. Annu. Rev. Immunol. 16:137-161.

41. Kawarada, Y., et al. 2001. NK- and CD8(+) T cellmediated eradication of established tumors by peritumoral injection of CpG-containing oligodeoxynucleotides. J. Immunol. 167:5247-5253.

42. Ballas, Z.K., et al. 2001. Divergent therapeutic and immunologic effects of oligodeoxynucleotides with distinct CpG motifs. J. Immunol. 167:4878-4886.

43. Vicari, A.P., et al. 2000. Antitumor effects of the mouse chemokine 6Ckine/SLC through angiostatic and immunological mechanisms. J. Immunol. 165:1992-2000.

44. Salcedo, R., et al. 2000. Human endothelial cells express CCR2 and respond to MCP-1: direct role of MCP-1 in angiogenesis and tumor progression. Blood. 96:34-40.

45. Lynch, D.H., et al. 1997. Flt3 ligand induces tumor regression and antitumor immune responses in vivo. Nat. Med. 3:625-631.

46. Favre-Felix, N., et al. 2000. Flt3 ligand lessens the growth of tumors obtained after colon cancer cell injection in rats but does not restore tumor-suppressed dendritic cell function. Int. J. Cancer. 86:827-834.

47. Chen, K., et al. 1997. Antitumor activity and immunotherapeutic properties of Flt3-ligand in a murine breast cancer model. Cancer Res. 57:3511-3516. 